\title{
Integrins In Mechanotransduction
}

\author{
Tyler D. Ross ${ }^{1}$, Brian G. Coon ${ }^{1}$, Sanguk Yun ${ }^{1}$, Nicolas Baeyens ${ }^{1}$, Keiichiro Tanaka ${ }^{1}$, \\ Mingxing Ouyang ${ }^{1}$, and Martin A. Schwartz ${ }^{1,2,3}$ \\ 1 Yale Cardiovascular Research Center, Section of Cardiovascular Medicine, Department of \\ Internal Medicine \\ 2 Department of Cell Biology
}

\section{Summary}

Forces acting on cells govern many important regulatory events during development, normal physiology, and disease processes. Integrin-mediated adhesions, which transmit forces between the extracellular matrix and the actin cytoskeleton, play a central role in transducing effects of forces to regulate cell functions. Recent work has led to major insights into the molecular mechanisms by which these adhesions respond to forces to control cellular signaling pathways. We briefly summarize effects of forces on organs, tissues, and cells; and then discuss recent advances toward understanding molecular mechanisms.

\section{Introduction}

The conversion of mechanical force into biochemical signals is critical in the development, physiology, and pathology of many, if not all tissues. Integrins play crucial roles, either as direct mechanotransducers, as transmitters of force to other elements, or as intermediates on pathways initiated by other receptors. In this review, we briefly summarize our current understanding of mechanotransduction, with emphasis on the role of integrins in these processes.

Effects of mechanical forces on organ physiology are widespread but especially significant for the cardiovascular system. Fluid shear stress from flowing blood is required for remodeling of the primitive yolk sac vascular plexus into a vascular tree [1] and for patterning the cardiac outflow tract [2]. In early lymphangiogenesis, interstitial fluid pressure stretches lymphatic endothelial cells, which stimulates integrin-dependent proliferation, and hence expansion of the lymphatics [3]. Furthermore, disturbed flow within the lymphatics initiates valve formation [4]. In heart disease, atherosclerotic plaques primarily occur at branch points and sharply curved regions of arteries where fluid flow patterns are disturbed [5]. Blood pressure stretches artery walls, which promotes vascular smooth muscle cell differentiation, and, if elevated, contributes to atherosclerosis [6]. Stretching cardiac myocytes by periodic contractions from the heart and increased resistance from blood pressure initially stimulates cardiac hypertrophy, which can progress to pathological heart failure [7].

\footnotetext{
(c) 2013 Elsevier Ltd. All rights reserved.

${ }^{3}$ Corresponding author: martin.schwartz@yale.edu, 300 George St., $7^{\text {th }}$ floor, New Haven CT 06511.
}

Publisher's Disclaimer: This is a PDF file of an unedited manuscript that has been accepted for publication. As a service to our customers we are providing this early version of the manuscript. The manuscript will undergo copyediting, typesetting, and review of the resulting proof before it is published in its final citable form. Please note that during the production process errors may be discovered which could affect the content, and all legal disclaimers that apply to the journal pertain. 
Outside the vasculature, mechanical loads from gravity and locomotion are essential for bone development and maintenance, and their absence is a major cause of osteoporosis. Compressive forces on bones are thought to trigger flow of interstitial fluid within small channels, which is sensed by osteocytes and governs bone remodeling [8]. Lung growth and physiology are regulated by stretching forces from inhalation [9]. The mechanics of the ECM (extracellular matrix) also regulates tissue function. ECM stiffness modulates breast cancer progression, where cancer cells promote stiffening of their environment, which feeds back to increase malignant behaviors such as loss of tissue architecture and invasion [10]. This type of positive feedback also operates in lung fibrosis [11] and atherosclerosis [12], where increased ECM stiffening promotes proliferation and ECM protein synthesis, which causes further stiffening

These organ level effects reflect events within cells. Fibroblasts on elastic substrata coated with ECM subject to biaxial cyclic stretch initially increase their rate of proliferation, but later become quiescent [13] and increase collagen type I production. Stretch also regulates cytoskeletal dynamics. During uniaxial cyclic stretch, actin stress fibers initially thicken along the axis of stretch; cells subsequently align to be either parallel or orthogonal to the force, depending on whether the frequency of stretch is slower or faster than the rate of cytoskeletal remodeling [14]. Integrins also mediate cellular responses to the rigidity or stiffness of the ECM, which controls many aspects of cell function; including proliferation, cytoskeletal remodeling, and migration [15]. This can also affect cell fate, such that mesenchymal stem cells will differentiate toward a certain lineage based on the stiffness of their matrix [16]. These effects are of considerable interest to tissue engineers who are using synthetic matrices to promote desired cell behaviors [17].

As might be expected, integrins are involved, one-way or another, in nearly all of these force-dependent processes. Work over the past several years has revealed a number of aspects of the mechanisms that govern integrin-mediated responses to externally applied forces transmitted through the ECM or endogenous forces modulated by ECM stiffness.

\section{Mechanisms Of Force Sensing}

One critical mechanosensory response that underlies cell behaviors is the strengthening of ligand-integrin-cytoskeleton linkages under force [18]. This process involves multiple mechanisms operating on different time scales. First, the integrins themselves undergo conformational changes under force. AFM studies of interactions between ICAM-1 and integrin aL $\beta 2$ integrin on live cells showed that binding is initially weak, but that pulling forces enhance transition rates to high affinity conformations that stabilize the integrinligand bond [19]. This behavior characterizes the linkage as a catch bond whose lifetime increases rather than decreases under force. Interestingly the increase of binding affinity is even more efficient under rapidly applied force, and is further enhanced under cyclic forces [20], both important points for rigidity sensing (below).

Tension also promotes the recruitment of cytoskeletal proteins that strengthen the integrinactin connection. One proposed mechanism involves talin, which binds directly to $\beta$ integrin cytoplasmic domains via its $\mathrm{N}$-terminal FERM domain, and to F-actin via its Cterminal rod domain. Vinculin binding sites within the rod are buried within helix bundles, but in vitro stretching of these domains exposes the binding sites [21]. Interestingly, imaging of a talin construct with $\mathrm{N}$ - and $\mathrm{C}$ - terminal fluorescent proteins in cells suggests that the molecule undergoes cyclic stretching [22]. Vinculin also binds F-actin and contributes to tension-induced adhesion stabilization [23]. Lastly, stretching cells or applying force to integrins using magnetic beads triggers the conversion of unoccupied low affinity integrins to the high affinity state, followed by their de novo binding to ECM proteins [24, 25]. In this mechanism, the bound integrins under tension signal to the unbound, low affinity integrins 
via a pathway that requires both TRPV4 and PI 3-kinase. These three mechanisms operate on time scales ranging from subseconds (integrin conformation), to seconds (cytoskeletal assembly), to multiple minutes (activation of other integrins).

Filamin A, another integrin and F-actin binding protein, is also a potential mechanotransducer. Filamin A also binds the Rac GTPase-accelerating protein, FilGAP. Tension across filamin in vitro strengthens its association with integrins and GPlb peptides $[26,27]$. However, strain on filamin A-crosslinked actin networks diminished affinity for FilGAP, which was released from the complex. Previous studies have demonstrated that cellular tension suppresses lammelipodial protrusion and global Rac activity in a FilGAPdependant manner [28]. Thus, filamin may mediate the known effects of tension on Rac activity by modulating FilGAP localization and function [29]. Signaling to RhoA is also an important aspect of adhesion strengthening. Force directly applied to integrins triggers Rho activation through the activation of two guanine nucleotide exchange factors (GEFs), LARG and GEF-H1 [30]. Modulation of Rho GTPases, at least in part through these mechanisms, provides another means by which forces regulate cytoskeletal architecture and cellgenerated tension.

The LIM domain protein Zyxin has also been implicated in mechanotransduction. Zyxin requires moderate force to localize to focal adhesions but at higher forces relocates to actin stress fibers and the nucleus [31]. This shift is mediated in part through Rho activation, which induces zyxin's MAPK-dependent phosphorylation. Zyxin also plays a key role in repairing broken actin filaments through the recruitment of VASP to free barbed ends of actin filaments [32]. Furthermore, nuclear zyxin participates in control of expression of genes for inflammation [33]. All together, it appears that zyxin mediates multiple responses to force, including stabilizing the cytoskeleton and inducing expression of genes that signal cell damage. These effects illustrate the spectrum of responses that depend on force magnitude.

\section{Rigidity Sensing}

As discussed above, cells also sense the mechanical properties of its ECM, so-called rigidity or stiffness sensing. These mechanisms share some features with sensing externally applied stretch. In this case, the origin of the force is the cells' own myosin, which is modulated by the substrate's mechanical properties. On soft ECM, myosin-dependent traction forces are decreased, resulting in smaller adhesions, reduced actin stress fibers, and less force across the ECM-integrin-cytoskeleton linkage [34]. In either setting, high forces result in large adhesions with high affinity integrins. The main focus of recent studies has been to elucidate the mechanisms by which cells read out ECM stiffness to control the applied force.

Rigidity sensing requires that cells first exert force on the substratum and read out the resultant strain or stress. Adhesions strengthen on stiff substrata but not soft, which subsequently influences cytoskeletal structure and signaling [35]. On rigid substrata, forces applied by the cell increase rapidly over a short distance, while soft surfaces deform so that force builds more slowly over a greater distance [36]. This is analogous to external force that is applied quickly or slowly, and similar mechanisms appear to operate under both conditions.

Periodic contractions within the lamellipodial cytoskeleton are observed during cell spreading [37], which could contribute to rigidity sensing. More recently, high-resolution traction force microscopy revealed that a subset of FAs (focal adhesions) undergo periodic fluctuations in traction that are modulated by substrate stiffness [38]. This dynamic behavior requires FAK, paxillin and vinculin, and is also important for durotaxis, the tendency of cells to migrate toward stiffer surfaces. These fluctuating forces are also likely to play into 
the recently observed effect where cyclic forces are especially effective at triggering conversion of low affinity integrins to the high affinity, long-lived state [20]. Taken together, these results suggest that force-dependent changes in integrin are an important component of rigidity sensing.

Stiffness sensing, however, also involves intracellular signaling. A siRNA screen of tyrosine kinases identified numerous components that affect specific rigidity responses [39]. Of particular interest, ERBB3 and TYRO3 abolished rigidity dependent differences in FA size and orientation, without affecting traction force. This result was surprising since it shows that force and FA structure can be uncoupled.

Recently, the mammalian Hippo pathway tumor suppressor proteins, YAP and TAZ, were found to mediate control of cell growth and differentiation by matrix rigidity [40, 41]. These proteins can localize to the nucleus where they interact with transcription factors and promote expression of a number of genes involved in cell growth and differentiation. Cell spreading and myosin-dependent contractility on rigid substrata promoted their nuclear localization, consistent with higher growth under these conditions. This pathway also appears to be crucial in control of stem cell differentiation and cell growth by matrix rigidity.

\section{Ion Channels and Integrins}

Stretching cells also increases lateral membrane tension, which activates mechanically gated ion channels, so-called stretch activated channels (SACs). Several recent studies identify unexpected links between integrins and SACs [42]. The gigantic mechanosensitive tetrameric cation channels, Piezo1 and Piezo2 [43], are SACs that allow calcium entry when activated but are distinct in their rapid deactivation. Interestingly, Piezos are related to Fam38 which is implicated in regulating integrin affinity [44]. Over-expressed Fam38 localized to the endoplasmic reticulum, where it regulated calcium release and promoted $\beta 1$ integrin conversion to the high affinity state. These studies did not examine stretch. However, stretch triggers conformational integrin activation [24], which appears to require calcium entry through TRPV4 channels that are activated when force is applied to integrins [25]. Whether these effects are related remains to be determined. Still, it is interesting that TRPV4 channel activity is facilitated by a rise of intracellular calcium through calciumcalmodulin binding [45]. This result provokes the speculation that Piezo channels might trigger a small, transient calcium spike that modulates TRP channel activity and downstream events.

Integrin-ion channel communication has also been observed in osteocytes. In this system, flow stimulated conformational activation of integrins $a 5 \beta 1$, which led to their interaction with connexin 43 hemichannels, results in hemichannel opening [46]. Curiously, this process did not require integrin binding to ECM. In this system, integrins may therefore play a role in mechanotransduction apart from their role as direct transducer.

\section{Measuring Tension}

Methods to measure tension across specific molecules are likely to find wide utility for these problems. These approaches all use constructs in which a fluorescence resonance energy transfer (FRET) donor is connected to an acceptor connected by an elastic element (Fig 2). Tension extends the "spring" to decrease FRET efficiency. The chemically synthesized sensors containing DNA springs were used to measuring extracellular forces [47], though these have not found much use. Results from genetically encoded sensors that measure force across $\mathrm{a}$-actinin and filamin suggested that tension within the cortical actin network is higher at the front of migrating cells [48, 49]. An experimentally calibrated spring based on 
the elastic sequence from spider silk incorporated into vinculin, revealed that average force across vinculin is around $2.5 \mathrm{pN} /$ molecule but is regulated, which was linked to adhesion strengthening [23]. This sensor was also used to measure force across E-cadherin [50]. Surprisingly, these investigators reported similar forces for plasma membrane E-cadherin both in and out of cell-cell junctions. However, studies on the endothelial cell-cell adhesion molecules VE-cadherin and PECAM-1 reported that force was only seen in the junctional pool [51]. Examination of effects of fluid shear stress revealed a complex sequence of events, which suggested that an upstream flow sensor triggers cytoskeletal rearrangements that alter force across the adhesion receptors. In summary, combining these methods with other cellular and biophysical methods offers the opportunity to elucidate mechanotransduction problems at an unprecedented level.

\section{Conclusions}

Past studies have identified a large array of physiological, pathological and cellular events that are controlled by mechanotransduction through integrin-dependent adhesions. More recent work has led to significant new insights into mechanisms that mediate these effects. Adhesion strengthening is one major mechanotransduction response by which forces trigger high affinity conformations in the integrins themselves, in addition to enhanced cytoskeletal linkages. Intracellular signaling pathways that mediate these effects as well as downstream changes in cellular growth and differentiation have been identified (Figure 1).

There remain, however, major unanswered questions. How do these immediate events such as changes in integrin conformation, channel opening, and cytoskeletal recruitment lead to downstream signaling events? Stretch and matrix stiffness trigger activation of kinases such as FAK, PI3K and src family kinases, and cytoplasmic retention of YAP/TAZ proteins, but how these events occur is largely unknown. How do integrins interact with ion channels at the molecular level? How do forces modulate the ongoing kinetics within adhesions that are intrinsically dynamic structures in which subunits constantly exchange and rearrange?

Further understanding will likely arise from the use of new technologies that allow for the precise measurement and application of force on living cells and visualization of molecular events. Methods that allow visualization of single [22] or small [52] numbers of molecules will continue to be crucial. Molecular tension sensors are also likely to play increasing roles in elucidating molecular mechanisms [23]. Improved, high resolution methods to apply forces to molecules in controlled ways, such as AFM [19, 20] and magnetic particles [30] will also be critical. Such techniques will facilitate detailed mapping of forces within cellular structures, subsequent changes in protein conformations and dynamics, and resultant transduction of information through cellular signaling pathways.

\section{Acknowledgments}

This work was supported by USPHS grant RO1 GM47214 to M.A.S.; Belgian American Education Foundation and WBI World excellence scholarships to N.B; and NIH training grant 5 T32 HL007950 to B.G.C.

\section{References}

1. Lucitti JL, Jones EAV, Huang C, et al. Vascular remodeling of the mouse yolk sac requires hemodynamic force. Development. 2007; 134:3317-26. [PubMed: 17720695]

2. Yashiro K, Shiratori H, Hamada H. Haemodynamics determined by a genetic programme govern asymmetric development of the aortic arch. Nature. 2007; 450:285-8. [PubMed: 17994097]

**3. Planas-Paz L, Strilić B, Goedecke A, et al. Mechanoinduction of lymph vessel expansion. EMBO J. 2012; 31:788-804. [PubMed: 22157817] [This study shows that the expansion of the 
embryonic lymphatic vasculature is stimulated by mechanical force from interstitial fluid pressure through $\beta 1$ integrins.]

*4. Sabine A, Agalarov Y, Maby-El Hajjami H, et al. Mechanotransduction, PROX1, and FOXC2 cooperate to control connexin 37 and calcineurin during lymphatic-valve formation. Dev Cell. 2012; 22:430-445. [PubMed: 22306086] [This paper demonstrates that lymphatic endothelial cells' initiate valvel formation in response to oscillatory fluid shear stress, through induction of the transcription factors PROX1 and FOXC2.]

5. Feaver RE, Gelfand BD, Wang C, et al. Atheroprone hemodynamics regulate fibronectin deposition to create positive feedback that sustains endothelial inflammation. Circ Res. 2010; 106:1703-11. [PubMed: 20378855]

6. Orr AW, Hastings NE, Blackman BR, Wamhoff BR. Complex regulation and function of the inflammatory smooth muscle cell phenotype in atherosclerosis. J Vasc Res. 2010; 47:168-180. [PubMed: 19851078]

7. Jaalouk DE, Lammerding J. Mechanotransduction gone awry. Nat Rev Mol Cell Biol. 2009; 10:6373. [PubMed: 19197333]

8. Rangaswami H, Schwappacher R, Marathe N, et al. Cyclic GMP and protein kinase G control a Srccontaining mechanosome in osteoblasts. Sci Signal. 2010; 3:ra91. [PubMed: 21177494]

9. Tschumperlin DJ, Boudreault F, Liu F. Recent advances and new opportunities in lung mechanobiology. J Biomech. 2010; 43:99-107. [PubMed: 19804885]

10. DuFort CC, Paszek MJ, Weaver VM. Balancing forces: architectural control of mechanotransduction. Nat Rev Mol Cell Biol. 2011; 12:308-319. [PubMed: 21508987]

11. Liu F, Mih JD, Shea BS, et al. Feedback amplification of fibrosis through matrix stiffening and COX-2 suppression. The Journal of Cell Biology. 2010; 190:693-706. [PubMed: 20733059]

**12. Kothapalli D, Liu S-L, Bae YH, et al. Cardiovascular Protection by ApoE and ApoE-HDL Linked to Suppression of ECM Gene Expression and Arterial Stiffening. Cell Rep. 2012; 2:1259-1271. [PubMed: 23103162] [This paper identified a lipid-independent function for ApoE-containing HDL that involves stimulating COX2 to suppress arterial smooth muscle matrix deposition, artery stiffening and atherosclerosis.]

13. Wang JH-C, Thampatty BP. An introductory review of cell mechanobiology. Biomech Model Mechanobiol. 2006; 5:1-16. [PubMed: 16489478]

14. Hoffman BD, Grashoff C, Schwartz MA. Dynamic molecular processes mediate cellular mechanotransduction. Nature. 2011; 475:316-323. [PubMed: 21776077]

15. Butcher DT, Alliston T, Weaver VM. A tense situation: forcing tumour progression. Nat Rev Cancer. 2009; 9:108-122. [PubMed: 19165226]

16. Engler AJ, Sen S, Sweeney HL, Discher DE. Matrix elasticity directs stem cell lineage specification. Cell. 2006; 126:677-89. [PubMed: 16923388]

17. Geiger B, Spatz JP, Bershadsky AD. Environmental sensing through focal adhesions. Nat Rev Mol Cell Biol. 2009; 10:21-33. [PubMed: 19197329]

18. Burridge K, Wittchen ES. The tension mounts: Stress fibers as force-generating mechanotransducers. J Cell Biol. 2013; 200:9-19. [PubMed: 23295347]

**19. Chen W, Lou J, Evans EA, Zhu C. Observing force-regulated conformational changes and ligand dissociation from a single integrin on cells. J Cell Biol. 2012; 199:497-512. [PubMed: 23109670] [This study provides evidence that applying force to a single aL $\beta 2$ integrin in live cells through its ligand ICAM-1 triggers conversion to a higher affinity, long-lived state, and characterized the dynamics.]

*20. Kong F, Li Z, Parks WM, et al. Cyclic Mechanical Reinforcement of Integrin-Ligand Interactions. Mol Cell. 2013 [This paper takes a further step beyond Chen et al to show that cyclic forces applied to an integrin fibronectin-a $5 \beta 1$ bond are especially effective at increasing integrin-ligand bond affinity and lifetime.]

21. del Rio A, Perez-Jimenez R, Liu R, et al. Stretching single talin rod molecules activates vinculin binding. Science. 2009; 323:638-641. [PubMed: 19179532]

*22. Margadant F, Chew LL, Hu X, et al. Mechanotransduction in vivo by repeated talin stretchrelaxation events depends upon vinculin. PLoS Biol. 2011; 9:e1001223. [PubMed: 22205879] [This study provides direct visual evidence that talin undergoes myosin-dependent cyclic stretch.] 
23. Grashoff C, Hoffman BD, Brenner MD, et al. Measuring mechanical tension across vinculin reveals regulation of focal adhesion dynamics. Nature. 2010; 466:263-266. [PubMed: 20613844]

24. Katsumi A, Naoe T, Matsushita T, et al. Integrin activation and matrix binding mediate cellular responses to mechanical stretch. J Biol Chem. 2005; 280:16546-16549. [PubMed: 15760908]

25. Thodeti CK, Matthews B, Ravi A, et al. TRPV4 channels mediate cyclic strain-induced endothelial cell reorientation through integrin-to-integrin signaling. Circ Res. 2009; 104:1123-30. [PubMed: 19359599]

**26. Ehrlicher AJ, Nakamura F, Hartwig JH, et al. Mechanical strain in actin networks regulates FilGAP and integrin binding to filamin A. Nature. 2011; 478:260-263. [PubMed: 21926999] [This study shows that the application of force across FLNA promotes binding to $\beta 7$ integrin while reducing binding to FilGAP.]

27. Rognoni L, Stigler J, Pelz B, et al. Dynamic force sensing of filamin revealed in single-molecule experiments. Proc Natl Acad Sci U S A. 2012; 109:19679-19684. [PubMed: 23150587]

28. Shifrin Y, Arora PD, Ohta Y, et al. The role of FilGAP-filamin A interactions in mechanoprotection. Mol Biol Cell. 2009; 20:1269-1279. [PubMed: 19144823]

29. Katsumi A, Milanini J, Kiosses WB, et al. Effects of cell tension on the small GTPase Rac. J Cell Biol. 2002; 158:153-64. [PubMed: 12105187]

**30. Guilluy C, Swaminathan V, Garcia-Mata R, et al. The Rho GEFs LARG and GEF-H1 regulate the mechanical response to force on integrins. Nat Cell Biol. 2011; 13:722-7. [PubMed: 21572419] [This study shows that tension applied to $\beta 1$ integrins causes the activation of LARG and GEF-H1, which increases RhoA activity.]

31. Hoffman LM, Jensen CC, Chaturvedi A, et al. Stretch-induced actin remodeling requires targeting of zyxin to stress fibers and recruitment of actin regulators. Mol Biol Cell. 2012; 23:1846-59. [PubMed: 22456508]

32. Chapin LM, Blankman E, Smith MA, et al. Lateral Communication between Stress Fiber Sarcomeres Facilitates a Local Remodeling Response. Biophys J. 2012; 103:2082-92. [PubMed: 23200042]

33. Anwar MA, Shalhoub J, Lim CS, et al. The effect of pressure-induced mechanical stretch on vascular wall differential gene expression. J Vasc Res. 2012; 49:463-78. [PubMed: 22796658]

34. Nemir S, West JL. Synthetic materials in the study of cell response to substrate rigidity. Ann Biomed Eng. 2010; 38:2-20. [PubMed: 19816774]

35. Moore SW, Roca-Cusachs P, Sheetz MP. Stretchy proteins on stretchy substrates: the important elements of integrin-mediated rigidity sensing. Dev Cell. 2010; 19:194-206. [PubMed: 20708583]

36. Chan CE, Odde DJ. Traction dynamics of filopodia on compliant substrates. Science. 2008; 322:1687-1691. [PubMed: 19074349]

37. Giannone G, Dubin-Thaler BJ, Döbereiner H-G, et al. Periodic Lamellipodial Contractions Correlate with Rearward Actin Waves. Cell. 2004; 116:431-443. [PubMed: 15016377]

*38. Plotnikov SV, Pasapera AM, Sabass B, Waterman CM. Force Fluctuations within Focal Adhesions Mediate ECM-Rigidity Sensing to Guide Directed Cell Migration. Cell. 2012; 151:1513-1527. [PubMed: 23260139] [This paper identifies a class of focal adhesions in which substrate traction undergoes temporal and spatial fluctuations, which correlates with the ability of cells to migrate toward stiffer substrates.]

*39. Prager-Khoutorsky M, Lichtenstein A, Krishnan R, et al. Fibroblast polarization is a matrixrigidity-dependent process controlled by focal adhesion mechanosensing. Nat Cell Biol. 2011; 13:1457-65. [PubMed: 22081092] [This paper conducts uses a protein tyrosine kinase siRNA screen to identify gene products required for various aspects of cell responses to substrate stiffness.]

**40. Dupont S, Morsut L, Aragona M, et al. Role of YAP/TAZ in mechanotransduction. Nature. 2011; 474:179-83. [PubMed: 21654799] [This paper shows that force-dependent regulation of the nuclear localization of the Hippo pathway transcription factors, YAP and TAZ, mediates substrate stiffness- and cell contractility-dependent regulation of cell differentiation and growth.]

*41. Wada K-I, Itoga K, Okano T, et al. Hippo pathway regulation by cell morphology and stress fibers. Development. 2011; 138:3907-3914. [PubMed: 21831922] [This paper shows that F-actin assembly inhibits the Hippo pathway through the reduction of Yap phosphorylation.] 
42. Katsumi A, Orr AW, Tzima E, Schwartz MA. Integrins in mechanotransduction. J Biol Chem. 2004; 279:12001-4. [PubMed: 14960578]

43. Coste B, Mathur J, Schmidt M, et al. Piezo1 and Piezo2 are essential components of distinct mechanically activated cation channels. Science. 2010; 330:55-60. [PubMed: 20813920]

44. McHugh BJ, Buttery R, Lad Y, et al. Integrin activation by Fam38A uses a novel mechanism of RRas targeting to the endoplasmic reticulum. J Cell Sci. 2010; 123:51-61. [PubMed: 20016066]

45. Strotmann R, Schultz G, Plant TD. Ca2+-dependent potentiation of the nonselective cation channel TRPV4 is mediated by a C-terminal calmodulin binding site. J Biol Chem. 2003; 278:26541-9. [PubMed: 12724311]

46. Batra N, Burra S, Siller-Jackson AJ, et al. Mechanical stress-activated integrin alpha5beta1 induces opening of connexin 43 hemichannels. Proc Natl Acad Sci U S A. 2012; 109:3359-64. [PubMed: 22331870]

47. Tarsa PB, Brau RR, Barch M, et al. Detecting force-induced molecular transitions with fluorescence resonant energy transfer. Angewandte Chemie (International ed. in English). 2007; 46:1999-2001. [PubMed: 17279589]

48. Meng F, Sachs F. Visualizing dynamic cytoplasmic forces with a compliance-matched FRET sensor. J Cell Sci. 2011; 124:261-269. [PubMed: 21172803]

49. Meng F, Suchyna TM, Sachs F. A fluorescence energy transfer-based mechanical stress sensor for specific proteins in situ. The FEBS journal. 2008; 275:3072-3087. [PubMed: 18479457]

50. Borghi N, Sorokina M, Shcherbakova OG, et al. E-cadherin is under constitutive actomyosingenerated tension that is increased at cell-cell contacts upon externally applied stretch. Proc Natl Acad Sci U S A. 2012; 109:12568-12573. [PubMed: 22802638]

51. Conway DE, Breckenridge MT, Hinde E, et al. Fluid Shear Stress on Endothelial Cells Modulates Mechanical Tension across VE-Cadherin and PECAM-1. Current biology : CB. 2013

52. Gardel ML, Schneider IC, Aratyn-Schaus Y, Waterman CM. Mechanical integration of actin and adhesion dynamics in cell migration. Annual review of cell and developmental biology. 2010; 26:315-333. 

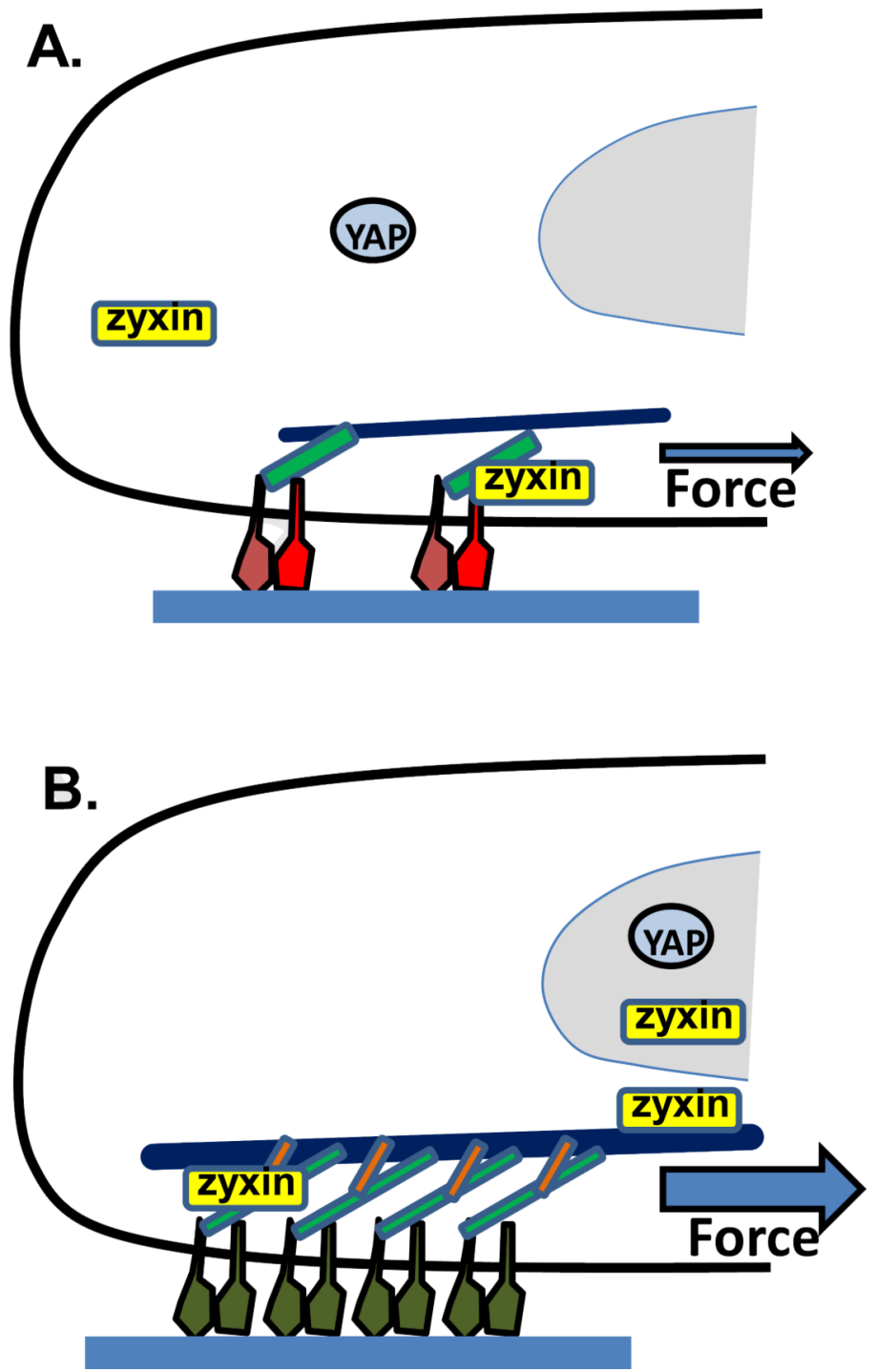

Figure 1. Partial summary of responses of integrin-mediated adhesions to force

Cells adhered to extracellular matrix (blue fabric) under (A) lower force or (B) high force. Force stimulates conversion of integrins from low affinity states (red) to more stable, high affinity states (green); stretching of talin (light green) to recruit vinculin (orange); also, additional integrins are activated and recruited to the adhesions. YAP (and TAZ) translocate to the nucleus to induce expression of cell cycle and differentiation genes. Zyxin localizes to focal adhesions under moderate force and at higher forces to actin stress fibers and the nucleus, where it promotes repair of the stress fibers and new gene expression. 

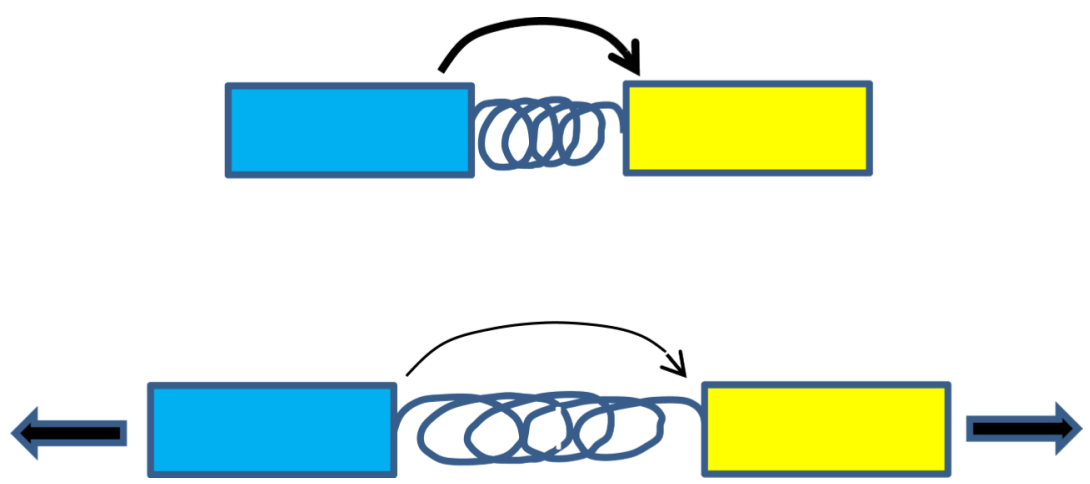

Figure 2. Molecular tension sensors

These methods all use a FRET pair, denoted here as blue and yellow fluorescent proteins, connected by an elastic element, denoted by a spring. Tension extends the spring, which decreased FRET efficiency. 